[20] 2. Die vom Kläger daneben geltend gemachten besonderen tatsächlichen und rechtlichen Schwierigkeiten der Rechtssache (§ 124 Abs. 2 Nr. 2 VwGO) sind im Zulassungsantrag weder hinreichend dargelegt worden, noch liegen sie vor.

[21] Der Kläger hat - wie oben ausgeführt - mit seiner Zulassungsbegründung nicht hinreichend dargelegt, welche begründeten Zweifel gegen die verwaltungsgerichtliche Entscheidung in tatsächlicher oder rechtlicher Hinsicht bestehen, die den Ausgang des Rechtsstreits als zumindest offen erscheinen lassen (vgl. Seibert in Sodan/Ziekow, Verwaltungsgerichtsordnung, 5. Aufl. 2018, $\S 124 a$ Rn. 209 und § 124 Rn. 106 ff.; Happ in Eyermann, VwGO, 15. Aufl. 2019, § 124a Rn. 68 und § 124 Rn. 27). Er hat auch nicht ausreichend verdeutlicht, weshalb der vom Verwaltungsgericht ermittelte Sachverhalt besonders unübersichtlich und die Sachverhaltsermittlung des Erstgerichts nicht ausreichend sein soll (vgl. Happ, a.a.O., § 124a Rn. 71). Der Hinweis allein auf den „umfangreichen Prozessstoff und die lange Prozessdauer" genügt den Darlegungsanforderungen jedenfalls nicht.
[22] Wie sich aus den obigen Ausführungen (unter 1.) ergibt, weist der vorliegende Fall weder von den maßgeblichen rechtlichen Grundlagen noch der anzustellenden Gefahrenprognose (gegenwärtige Gefahr i.S.v. Art. 25 PAG a.F.) her komplexe oder noch nicht geklärte Rechtsfragen auf.

[23] Eine ausführliche und auf alle aufgeworfenen Einzelfragen bzw. Einwendungen eingehende Begründung des angefochtenen Urteils allein ist noch kein entscheidendes Indiz für das Vorliegen besonderer Schwierigkeiten der Streitsache.

[24] Die Kostenentscheidung folgt jeweils aus § 154 Abs. 2 VwGO.

[25] Die Streitwertfestsetzung für die Zulassungsverfahren beruht auf $\S 63$ Abs. 2 Satz 1, § 47 Abs. 1 und 3 und $\S 52$ Abs. 1 GKG.

[26] Dieser Beschluss ist unanfechtbar ( $\$ 152$ Abs. 1 VwGO). Mit der Ablehnung des Antrags auf Zulassung der Berufung wird die Entscheidung des Verwaltungsgerichts rechtskräftig (§ 124a Abs. 5 Satz 4 VwGO).

\title{
Streit um Sitz im Stiftungsrat des „Fürst-Pückler-Museum Park und Schloss Branitz"
}

VerfGBbg, Beschluss vom 16. April 2021 - VfGBbg 71/18

\section{Tenor}

$\S 7$ Abs. 1 Satz 2 des Gesetzes über die Errichtung der Stiftung „Fürst-Pückler-Museum Park und Schloss Branitz" (SFPMG) vom 14. Dezember 2017 (GVBI I Nr. 31) verletzt den Beschwerdeführer in seinem Grundrecht auf Gleichbehandlung aus Art. 12 Abs. 1 Satz 1 und Satz 2 Verfassung des Landes Brandenburg und ist nichtig.

Im Übrigen wird die Verfassungsbeschwerde teils verworfen, teils als unbegründet zurückgewiesen.

Das Land Brandenburg hat dem Beschwerdeführer seine notwendigen Auslagen für das Verfassungsbeschwerdeverfahren zu erstatten.

\section{Gründe}

\section{A}

[1] Der Beschwerdeführer wendet sich gegen eine gesetzliche Regelung zur Beteiligung der Erben des Fürsten Pückler im Stiftungsrat der Stiftung „Fürst-Pückler-Museum Park und Schloss Branitz“.
I.

[2] Der Beschwerdeführer ist zu einem Anteil von einem Zwölftel Mitglied einer ursprünglich aus sieben Personen bestehenden Erbengemeinschaft nach Theodora Gräfin von Pückler, geb. Gräfin zu Limburg-Styrum, die ihr Alleinerbe an Schloss und Park Branitz auf den 1871 kinderlos verstorbenen Hermann Ludwig Heinrich Fürst von Pückler-Muskau zurückführte und die im Zuge der Bodenreform 1946 auf besatzungsrechtlicher Grundlage enteignet wurde.

[3] Der Erbengemeinschaft nach Theodora Gräfin von Pückler wurden mit Bescheid des Landesamts zur Regelung offener Vermögensfragen vom 30. Oktober 2008 konkret bezeichnete 1.746 bewegliche Vermögensgegenstände zurückübertragen. Der Bescheid stellte den unentgeltlichen öffentlichen Nießbrauch der Stadt Cottbus an den Vermögensgegenständen gemäß $\S 5$ Abs. 2 Satz 1 Ausgleichsleistungsgesetz (AusglLeistG) bis zum 1. Dezember 2014 fest. Mit Bescheid des Landesamts zur Regelung offener Vermögensfragen vom 24. September 2014 wurden der Erbengemeinschaft nach Theodora Gräfin von Pückler konkret bezeichnete 4.673 bewegliche Vermögensgegenstände der Pückler-Callenberg-Bibliothek Branitz, die in die Landesbibliothek Potsdam überführt worden waren, zurückübertragen und der unentgeltliche öffentliche Nießbrauch gemäß § 5 Abs. 2 Satz 1 AusglLeistG bis zum 1. Dezember 2014 festgestellt. 
[4] Der Landtag beschloss am 14. Dezember 2017 das zum 1. Januar 2018 in Kraft getretene Gesetz über die Errichtung der rechtsfähigen Stiftung des öffentlichen Rechts „FürstPückler-Museum Park und Schloss Branitz" (SFPMG). Diese Stiftung trat an die Stelle der nicht-rechtsfähigen kommunalen Stiftung der Stadt Cottbus „Fürst-Pückler-Museum Park und Schloss Branitz".

[5] Mitglied im Stiftungsrat der kommunalen Stiftung war Sylvius Hermann Graf von Pückler. Der im Jahr 2017 verstorbene Sylvius Hermann Graf von Pückler war Mitglied der Erbengemeinschaft nach Theodora Gräfin von Pückler, und zwar als ein Sohn des Adrian Heinrich Kurt Günther Georg Graf von Pückler (19051945). Der Beschwerdeführer ist ein Enkel des Carl Erdmann Victor Hans Theodor Heinrich Graf von Pückler (1906-1941), der ein Bruder des Adrian Graf von Pückler war. Beide sind Söhne der Theodora Gräfin von Pückler und des 1937 verstorbenen August Karl Heinrich Georg Graf von Pückler. Die Erbengemeinschaft nach Theodora Gräfin von Pückler besteht nur noch aus den Nachkommen ihrer Söhne Adrian Graf von Pückler und Carl Erdmann Graf von Pückler. [...]

[6] Das Eigentum an den Liegenschaften des Denkmalensembles Park und Schloss Branitz ging mit dem Gesetz zur Errichtung der Stiftung von der Stadt Cottbus auf die Stiftung über. Die Landesstiftung betreut die an die Erbengemeinschaft nach Theodora Gräfin von Pückler zurückübertragene und in das Schloss Branitz zurücküberführte Pückler-Callenberg-Bibliothek und die weiteren sonstigen an die Erbengemeinschaft nach Theodora Gräfin von Pückler zurückübertragenen Vermögensgegenstände, an denen bis zum 1. Dezember 2014 ein öffentlicher Nießbrauch bestand. Nach Angaben des Ministeriums der Justiz gehören zu den zurückübertragenen Vermögensgegenständen auch Archivalien des Gutsarchivs. Nach Angaben des Beschwerdeführers hat die Stadt Cottbus das Recht aus $\S 5$ Abs. 2 Satz 2 AusglLeistG nicht ausgeübt und sind die Konditionen der weiteren Leihgabe zwischen der Stiftung und den Eigentümern, d.h. der Erbengemeinschaft nach Theodora Gräfin von Pückler, bislang nicht geregelt worden.

[7] Gesetzlich festgelegter Zweck der Stiftung „Fürst-PücklerMuseum Park und Schloss Branitz" ist die Erhaltung, Pflege, Erforschung, Erschließung und Präsentation des Gesamtkunstwerks aus Garten- und Landschaftsgestaltung, Architektur, Raumausstattung und der Museumssammlungen des Fürsten Hermann von Pückler-Muskau in Branitz. Dazu gehören unter anderem die Pflege und der Ausbau der Museumssammlungen sowie die digitale Erschließung und Vermittlung der Sammlungsobjekte.

[8] Dem Stiftungsrat gehören gemäß § 7 Abs. 1 Satz 1 Nr. 1 bis 6 SFPMG notwendig acht Mitglieder an, die Vertreter des Landes, der Stadt Cottbus und des Bundes sind. Der vom Beschwerdeführer angegriffene $§ 7$ Abs. 1 Satz 2 SFPMG lautet:
„Die Erben nach Adrian Heinrich Kurt Günther Georg Graf von Pückler Freiherr von Groditz, geboren am 24. März 1905, gestorben am 19. Januar 1945, sind berechtigt, eine Vertreterin oder einen Vertreter in den Stiftungsrat zu entsenden."

Hierzu heißt es in der Begründung des Gesetzes (Zu § 7, Zu Absatz 1, LT-Drs. 6/7369, S. 5):

„Im Zuge der Regelungen des Entschädigungs- und Ausgleichsleistungsgesetzes aus dem Jahr 1994 erhielt die aus sieben Personen bestehende Erbengemeinschaft der Grafen Pückler die erhalten gebliebenen beweglichen Kunst- und Kulturgüter, die Pückler-Callenberg Bibliothek und Archivalien des Gutsarchivs zurückübertragen. Auch nach der Rückübertragung blieben diese ohne Unterbrechung der Öffentlichkeit und der wissenschaftlichen Forschung im Schloss Branitz zugänglich. Ergänzend hierzu wurden dem Fürst-Pückler-Museum von Hermann Sylvius Graf von Pückler, ältester Sohn von Adrian Heinrich Kurt Günther Georg Graf von Pückler Freiherr von Groditz (im Folgenden Adrian von Pückler genannt), Leihgaben aus dem privaten Besitz zur Verfügung gestellt. Zudem engagierten und engagieren er und seine Angehörigen sich für die Erhaltung des Pücklerschen Gesamterbes in Park und Schloss Branitz. In der Konsequenz diesen Engagements wurde Hermann Sylvius Graf von Pückler durch einen Beschluss der Stadtverordnetenversammlung Cottbus als Vertreter der Familie nach Adrian von Pückler als Mitglied in den Stiftungsrat der kommunalen unselbständigen Stiftung „Fürst-Pückler-Museum Park und Schloss Branitz" berufen. Angesichts der Wichtigkeit des Verbleibs der Sammlungen in Park und Schloss Branitz und mit Blick auf das weitere gewünschte Engagement der Erben nach Adrian von Pückler für Branitz soll diesen zugestanden und angeboten werden, eine Vertreterin oder einen Vertreter in den Stiftungsrat der öffentlich-rechtlichen Stiftung zu entsenden."

Die weiteren gesetzlichen Regelungen sehen vor, dass gemäß $\S 7$ Abs. 5 Satz 2 SFPMG nach näherer Maßgabe der Satzung weitere Personen vom Stiftungsrat beratend zu den Stiftungsratssitzungen hinzugezogen werden können. Der Stiftungsrat hat gemäß § 8 SFPMG Satzungs- und Haushaltsrecht, beschließt über die grundsätzlichen Angelegenheiten der Stiftung und legt die wesentlichen Aufgaben und Tätigkeiten der Stiftung fest. Er entscheidet insbesondere über alle Geschäfte, die nicht nach § 10 SFPMG dem Vorstand obliegen. Unter anderem stehen gemäß § 8 Abs. 4 Nr. 3 SFPMG die Annahme von Erbschaften und gemäß Nr. 4 die Veräußerung von Sammlungsgegenständen unter dem Vorbehalt der Zustimmung des Stiftungsrats, wobei eine Veräußerung nur zulässig ist, wenn mit dem Veräußerungserlös der Sammlungsbestand ergänzt wird. Gemäß § 9 Abs. 2 Satz 2 SFPMG werden Beschlüsse des Stiftungsrats mit der Stimmenmehrheit der Anwesenden gefasst.

\section{II.}

[9] Der Beschwerdeführer hat am 27. Dezember 2018 Verfassungsbeschwerde erhoben. 
[10] Er trägt vor, $\S 7$ Abs. 1 Satz 2 SFPMG verstoße gegen Art. 3 Abs. 3 Grundgesetz (GG) und Art. 12 Abs. 1 und Abs. 2 Verfassung des Landes Brandenburg (LV). Niemand dürfe aufgrund seiner Herkunft oder Abstammung benachteiligt oder bevorzugt werden. Die Sozialbindung des Eigentums aus Art. 14 Abs. 2 GG und Art. 41 Abs. 2 LV verpflichte den Gesetzgeber, einen Rahmen für die Mitwirkung der Eigentümer zu schaffen. Nach § 7 Abs. 1 Satz 2 SFPMG werde nicht der gesamten Erbengemeinschaft, sondern lediglich einzelnen ihrer Mitglieder das Recht eingeräumt, im Stiftungsrat mitzuwirken. Er selbst werde von der Berechtigung zur Mitwirkung im Stiftungsrat und von Auskunftsrechten ausgeschlossen. Für die Ungleichbehandlung gebe es keinen sachlichen Grund von solcher Art und solchem Gewicht, dass er die Ungleichbehandlung rechtfertigen könne.

[11] Dass der verstorbene Sylvius Hermann Graf von Pückler dem Museum weitere Leihgaben aus seinem privaten Besitz zur Verfügung gestellt habe, könne nicht die Bevorzugung aller Erben nach Adrian von Pückler, d. h. auch der Geschwister, deren Erben und der Erben des Sylvius Hermann Graf von Pückler gegenüber den übrigen Miterben begründen. Die privaten Leihgaben von Sylvius Hermann Graf von Pückler stünden in keinem Verhältnis zur Leihgabe der gesamten Erbengemeinschaft und seien überdies jederzeit widerrufbar. Allenfalls die Ehefrau des Sylvius Hermann Graf von Pückler habe sich noch für Schloss und Park Branitz engagiert. Die Geschwister des Sylvius Hermann Graf von Pückler hätten sich lediglich zu repräsentativen Anlässen in Branitz eingefunden. Es sei auch nicht das Verdienst von Sylvius Hermann Graf von Pückler, dass die an die Erbengemeinschaft nach Theodora Gräfin von Pückler restituierten Sammlungen nach 2014 im Schloss Branitz geblieben seien. Dafür seien alle Mitglieder der Erbengemeinschaft gewesen. Alle Erben unterstützten auch das Ausleihen von Gegenständen an andere Museen und Ausstellungen. Der Beschwerdeführer unterstütze das Anliegen seines 1937 verstorbenen Urgroßvaters, der testamentarisch verfügt habe, dass das Erbe niemals geteilt werden dürfe. Er habe kein Interesse daran, den Stiftungsrat zu blockieren. Dies sei aufgrund des Stimmenverhältnisses auch ausgeschlossen.

[12] Nachdem er von dem Gesetzentwurf durch Zufall erfahren habe, habe er seine Bedenken gegen die Verfassungsmäßigkeit des § 7 Abs. 1 Satz 2 SFPMG dem Landtag Brandenburg schriftlich mitgeteilt. Eine Antwort habe er nicht erhalten.

[13] Der Beschwerdeführer beantragt,

die Unwirksamkeit von § 7 Abs. 1 Gesetz über die Errichtung der Stiftung „Fürst-Pückler-Museum Park und Schloss Branitz" (SFPMG) vom 14. Dezember 2017 (GVBI I Nr. 31) festzustellen.
III.

[14] Der Landtag Brandenburg, die Landesregierung, die Stiftung und die vom Beschwerdeführer benannten Mitglieder der Erbengemeinschaft haben Kenntnis von dem Verfahren beziehungsweise Gelegenheit zur Stellungnahme erhalten.

\section{IV.}

[15] Die Ministerin der Justiz trägt für die Landesregierung vor, dass die Kriterien der Abstammung oder Herkunft im Sinne des absoluten Differenzierungsverbots des Art. 12 Abs. 2 LV nicht Grund für die Regelung in $\S 7$ Abs. 1 Satz 2 SFPMG gewesen seien. Ausschließlicher Anknüpfungspunkt für die unterschiedliche Behandlung des Beschwerdeführers und der Erben nach Adrian Graf von Pückler sei vielmehr der persönliche Einsatz des Sylvius Hermann Graf von Pückler und seiner Familie. Sylvius Hermann Graf von Pückler habe mit seinen Geschwistern, den anderen Kindern des Adrian Graf von Pückler, bis 1945 selbst in Schloss Branitz gelebt. Er habe sich nach der Wiedervereinigung sehr intensiv für die Herstellung des Branitzer Parks, die Sanierung der Gebäude und die Kunst- und Kulturgüter eingesetzt. Zudem habe er Forschungen angestellt, um infolge der Ereignisse des Zweiten Weltkriegs aus dem Familienbesitz abhandengekommene oder verbrachte Bücher, Gemälde und Mobiliar wieder aufzuspüren und sie wenn möglich anzukaufen. Auch nach der Rückübertragung der erhalten gebliebenen beweglichen Kunst- und Kulturgüter, der Pückler-Callenberg Bibliothek und der Archivalien des Gutsarchivs an die Erbengemeinschaft seien diese ohne Unterbrechung der Öffentlichkeit und der wissenschaftlichen Forschung im Schloss Branitz zugänglich geblieben, was vor allem das Verdienst von Sylvius Hermann Graf von Pückler gewesen sei. Wiederholt habe dieser dem Fürst-Pückler-Museum zudem Leihgaben aus seinem privaten Besitz zur Verfügung gestellt. Überdies habe er Teile des ehemals zum Pücklerschen Gesamtbesitz gehörenden unbeweglichen Erbes zurückgekauft, das stark renovierungsbedürftige Parkinspektorenhaus im Branitzer Park nahe des Schlosses sowie den Branitzer Kindergarten saniert, örtliche Betriebe reorganisiert und einen eigenen Forstbetrieb in Cottbus und Bagenz aufgebaut. In der Konsequenz dieses Engagements sei Sylvius Hermann Graf von Pückler durch einen Beschluss der Stadtverordnetenversammlung von Cottbus als Vertreter der Familie nach Adrian Graf von Pückler in den Stiftungsrat der kommunalen unselbständigen Stiftung „FürstPückler-Museum Park und Schloss Branitz" berufen worden, wo er an der Entwicklung des Gesamtstandorts und dem dortigen Verbleib der Sammlung bis zu seinem Tod mitgewirkt habe. Außerdem habe er sich aktiv im Kuratorium der Branitzer Stiftung sowie im internationalen Fachbeirat, dem „International Conservation Board", der alle drei Pückler-Parkverwaltungen, Branitz sowie die deutsche und die polnische Parkverwaltung Bad Muskau-Muzakowski, angehörten, engagiert. Aufgrund der vorgenannten persönlichen Leistungen seien Sylvius Hermann Graf von Pückler Verdienstorden des Landes Brandenburg und der Bundesrepublik Deutschland zuerkannt worden. [...] Die 
Regelung in $\S 7$ Abs. 1 Satz 2 SFPMG verstoße auch nicht gegen den allgemeinen Gleichheitssatz aus Art. 12 Abs. 1 LV. Die gesetzliche Differenzierung sei aus den genannten Gründen sachgerecht. Durch die Regelung solle zudem verhindert werden, dass immer noch währende Erbstreitigkeiten innerhalb der Erbengemeinschaft nach Theodora Gräfin von Pückler in den Stiftungsrat getragen würden. Andernfalls sei eine Blockierung und Behinderung der Arbeit im Stiftungsrat und dessen Funktionsunfähigkeit zu befürchten. Den Erben nach Adrian Graf von Pückler gehöre ein Anteil von 10/12 der Erbengemeinschaft nach Theodora Gräfin von Pückler. Aufgrund der privaten Leihgaben des Sylvius Hermann Graf von Pückler und seiner Familie könne die Stiftung ihren Zweck auch ohne das Eigentum der Erbengemeinschaft nach Theodora Gräfin von Pückler erfüllen. $\S 7$ Abs. 1 Satz 2 SFPMG berühre nicht das Eigentumsrecht aus Art. 41 LV. Die wirksame Teilnahme an Entscheidungen über das Gesamthandsvermögen der Erbengemeinschaft, den Erhalt der benötigten Informationen und mögliche Auseinandersetzungen mit den Miterben oder der Stiftung könne der Beschwerdeführer auf dem Rechtsweg durchsetzen.

\section{B.}

[16] 1. Die Verfassungsbeschwerde ist zulässig, soweit sie sich gegen $§ 7$ Abs. 1 Satz 2 SFPMG richtet. Im Übrigen ist sie unzulässig.

[17] a) Das Gesetz über die Errichtung der Stiftung „FürstPückler-Museum Park und Schloss Branitz" (SFPMG) vom 14. Dezember 2017 (GVBI I Nr. 31) ist als Akt der öffentlichen Gewalt des Landes Brandenburg gemäß § 45 Abs. 1, § 46 Verfassungsgerichtsgesetz Brandenburg (VerfGGBbg) ein statthafter Gegenstand der Verfassungsbeschwerde, vgl. § 47 Abs. 3, $\S 50$ Abs. 4 Satz 1 VerfGGBbg.

[18] b) Die Verfassungsbeschwerde ist gemäß $\S 47$ Abs. 3 VerfGGBbg binnen eines Jahres nach dem Inkrafttreten des Gesetzes erhoben worden.

[19] c) Der Grundsatz der Subsidiarität steht der Zulässigkeit der Verfassungsbeschwerde nicht entgegen. Der Beschwerdeführer kann die angestrebte Einbeziehung in den Kreis der Berechtigten in $\S 7$ Abs. 1 Satz 2 SFPMG nicht vor den Fachgerichten erreichen. Der Gesetzgeber ist auf seine Einwendungen im Gesetzgebungsverfahren nicht eingegangen. Dass sich der Beschwerdeführer anscheinend nicht dagegen gewandt hat, dass Sylvius Hermann Graf von Pückler einen Sitz im Stiftungsrat der kommunalen Stiftung innehatte, führt nicht zur Unzulässigkeit der Verfassungsbeschwerde aus Gründen der Subsidiarität. Die Situation aufgrund des Gesetzes ist eine andere als zu Zeiten der kommunalen Stiftung. Die Stadt Cottbus hatte Sylvius Hermann Graf von Pückler einen Sitz im Stiftungsrat aufgrund seiner Leistungen für die Stiftung angeboten. § 7 Abs. 1 Satz 2 SFPMG erweitert jedoch nach dem Tod von Sylvius Hermann Graf von Pückler den Kreis der Kandidaten für einen Sitz im Stiftungsrat der Landesstiftung auf Geschwister des Sylvius Hermann Graf von Pückler und weitere Erben nach Adrian Graf von Pückler, während andere Mitglieder der leihgebenden Erbengemeinschaft nach Theodora Gräfin von Pückler davon jetzt ausdrücklich und auf Dauer ausgeschlossen sind.

[20] d) Der Beschwerdeführer ist auch hinsichtlich des $\S 7$ Abs. 1 Satz 2 SFPMG beschwerdebefugt. Die Beschwerdebefugnis setzt die Möglichkeit voraus, selbst, unmittelbar und gegenwärtig in einer grundrechtlich geschützten Rechtsposition beeinträchtigt bzw. verletzt zu sein (st. Rspr., vgl. Beschlüsse vom 15. Dezember 2017 - VfGBbg 63/16 -, m.w. N., und vom 9. Oktober 2015 - VfGBbg 8/15 EA -, https://verfassungsgericht. brandenburg.de).

[21] Die Beschwerdebefugnis hinsichtlich eines Verstoßes gegen den Gleichheitsgrundsatz setzt dabei den Vortrag voraus, dass es durch die gesetzliche Regelung zu einer unterschiedlichen Behandlung miteinander vergleichbarer Personengruppen und hierdurch zu einem konkret darzustellenden Nachteil, d.h. zu einer Beeinträchtigung eines rechtlich geschützten Interesses bei einer der Gruppen kommt, ohne dass die Ungleichbehandlung durch hinreichende Sachgründe gerechtfertigt ist (vgl. Beschluss vom 9. September 2016 - VfGBbg 13/16 -, https:// verfassungsgericht.brandenburg.de), und der Beschwerdeführer dadurch selbst benachteiligt wird.

[22] aa) Der Beschwerdeführer legt auch die Möglichkeit dar, durch den gesetzlichen Ausschluss in $\S 7$ Abs. 1 Satz 2 SFPMG selbst, unmittelbar und gegenwärtig in seinem Gleichbehandlungsanspruch verletzt zu sein. Er zeigt in der Beschwerdeschrift schlüssig die Möglichkeit auf, dass er durch den Ausschluss von dem Recht in $\S 7$ Abs. 1 Satz 2 SFPMG, einen Vertreter in den Stiftungsrat zu entsenden oder selbst entsandt zu werden, in der Wahrnehmung seiner Interessen gegenüber der Stiftung, die die zurückübertragenen Sammlungen der Erbengemeinschaft nach Theodora Gräfin von Pückler betreut, als Mitglied der Erbengemeinschaft gegenüber den anderen Erben nach Adrian Graf von Pückler benachteiligt werden und dadurch sein Recht auf Gleichbehandlung aus Art. 12 Abs. 1 und Abs. 2 LV verletzt sein könnte.

[23] bb) Keine Beschwerdebefugnis besteht jedoch hinsichtlich des vom Antrag mit umfassten $\S 7$ Abs. 1 Satz 1 SFPMG. Die Beschwerdeschrift verhält sich nicht dazu und zeigt daher nicht die Möglichkeit auf, dass durch die gesetzliche Regelung der übrigen Zusammensetzung des Stiftungsrats gemäß $\S 7$ Abs. 1 Satz 1 SFPMG Rechte des Beschwerdeführers aus der Landesverfassung verletzt werden können. Bezüglich $\S 7$ Abs. 1 Satz 1 SFPMG ist die Verfassungsbeschwerde daher gemäß § 21 Satz 1 VerfGGBbg als unzulässig zu verwerfen.

[24] cc) Soweit der Beschwerdeführer neben Art. 12 Abs. 1 und Abs. 2 LV die Verletzung von Art. 3 Abs. 3 GG benennt, legt er nicht die Möglichkeit einer Verletzung in Grundrechten der Landesverfassung dar, da es sich bei Art. 3 Abs. 3 GG nicht um ein in der Landesverfassung gewährtes Grundrecht handelt (vgl. Beschluss vom 16. August 2019 - VfGBbg 41/19 -, https://verfas- 
sungsgericht.brandenburg.de). Die Rüge des Art. 3 Abs. 3 GG ist daher auch gemäß $§ 21$ Satz 1 VerfGGBbg als unzulässig zu verwerfen. Sofern der Beschwerdeführer vorträgt, die Sozialbindung des Eigentums aus Art. 14 Abs. 2 GG und Art. 41 Abs. 2 LV verpflichte den Gesetzgeber, einen Rahmen für die Mitwirkung der Eigentümer zu schaffen, wird dies als Argument des Beschwerdeführers im Hinblick auf eine gesetzliche Neuregelung verstanden, dass die Erbengemeinschaft in der Stiftung nicht unberücksichtigt bleiben könne, nicht jedoch dahingehend, dass die Verletzung des Art. 41 LV gerügt wird.

[25] e) Der Beschwerdeführer hat auch ein Rechtsschutzbedürfnis für eine Verfassungsbeschwerde.

[26] aa) Das Rechtsschutzbedürfnis für die Verfassungsbeschwerde entfällt nicht dadurch, dass der Beschwerdeführer theoretisch andere Mitwirkungsmöglichkeiten bei der Stiftung hätte, indem er etwa als weitere beratende Person gemäß $\S 7$ Abs. 5 Satz 2 SFPMG an Sitzungen des Stiftungsrats teilnehmen könnte. Denn diese Möglichkeit gewährt kein Recht, als Vertreter der Erbengemeinschaft entsandt zu werden, kein Stimmrecht und kein Mitwirkungsrecht bei der Bestimmung eines Vertreters der Erbengemeinschaft, keine Vertretung im Stiftungsrat durch den Vertreter mit Stimmrecht sowie keine regelmäßige Teilnahme an den Sitzungen des Stiftungsrats.

[27] bb) Das Rechtsschutzbedürfnis entfällt auch nicht dadurch, dass der Beschwerdeführer möglicherweise nur geringe Chancen hat, selbst als Vertreter der Erbengemeinschaft in den Stiftungsrat entsandt zu werden. Denn es geht ihm auch um sein Mitwirkungsrecht an der Bestimmung eines Vertreters und die gleiche formale Berechtigung als solche. Schließlich können sich auch die Mehrheiten und Interessen in der Erbengemeinschaft ändern. Zudem könnte der Gesetzgeber unterschiedliche Neuregelungen treffen, zum Beispiel auch den Erben nach Carl Erdmann Graf von Pückler einen weiteren Sitz im Stiftungsrat einräumen.

[28] 2. Die hinsichtlich § 7 Abs. 1 Satz 2 SFPMG zulässige Verfassungsbeschwerde ist überwiegend begründet. Die Vorschrift verstößt gegen Art. 12 Abs. 1 LV, jedoch nicht gegen Art. 12 Abs. 2 LV.

[29] a) Die vom Gesetzgeber getroffene Differenzierung innerhalb der Eigentümer-Erbengemeinschaft nach Theodora Gräfin von Pückler zwischen den Erben nach Adrian Graf von Pückler und den übrigen Erben unterfällt nicht dem absoluten Diskriminierungsverbot nach dem Merkmal der Abstammung im Sinne des Art. 12 Abs. 2, 1. Fall LV oder nach dem Merkmal der sozialen Herkunft oder Stellung im Sinne des Art. 12 Abs. 2, 6. Fall LV.

[30] aa) Nach der gegenüber Art. 12 Abs. 1 LV speziellen Vorschrift in Art. 12 Abs. 2 LV darf niemand wegen seiner Abstammung, Nationalität, Sprache, des Geschlechts, der sexuellen Identität, sozialen Herkunft oder Stellung, einer Behinderung, der religiösen, weltanschaulichen oder politischen Überzeugung oder aus rassistischen Gründen bevorzugt oder benachteiligt werden. Der Schutzbereich dieser Norm ist betroffen, wenn eine Ungleichbehandlung anhand der genannten Differenzierungskriterien erfolgt und zu einer Benachteiligung führt. Das ist hier nicht der Fall. Die Regelung des $\S 7$ Abs. 1 Satz 2 SFPMG, wonach "die Erben nach Adrian Heinrich Kurt Günther Georg Graf von Pückler Freiherr von Groditz, geboren am 24. März 1905, gestorben am 19. Januar 1945, berechtigt sind, eine Vertreterin oder einen Vertreter in den Stiftungsrat zu entsenden", knüpft ausdrücklich an die Erbenstellung nach Adrian Graf von Pückler an. Die Stellung als Erbe ist allerdings weder gleichbedeutend mit der Abstammung im Sinne des Art. 12 Abs. 2, 2. Fall LV noch mit der sozialen Herkunft oder Stellung im Sinne des Art. 12 Abs. 2, 6. Fall LV. Abstammung ist die natürliche biologische Beziehung eines Menschen zu seinen Vorfahren (vgl. BVerfG, Beschluss vom 22. Januar 1959 - 1 BvR 154/55 -, BVerfGE 9, 124, 128, juris). Die Abstammung ist insoweit in legitimer Weise teleologisch einschränkend auszulegen und von der Erbenstellung zu unterscheiden, auch wenn beides bei der Erbfolge zusammenfallen kann (vgl. dazu Nußberger, in: Sachs, 9. Aufl. 2021, GG Art. 3 Rn. 292 f.). Erbe ist eine Person beziehungsweise Erben sind mehrere Personen, auf die mit dem Tode einer anderen Person deren Vermögen als Ganzes übergeht, § 1922 Bürgerliches Gesetzbuch (BGB). Abkömmlinge des Erblassers, d. h. die Kinder und weiteren Nachkommen eines Menschen, sind zwar gesetzliche Erben erster Ordnung, § 1924 Abs. 1 BGB, was im Erbrecht über die biologische Abstammung hinaus auch die in der Regel unproblematische rechtlich anerkannte Abstammung gemäß §§ 1591, 1592 BGB voraussetzt. So ist zum Beispiel auch der überlebende Ehegatte gesetzlicher Erbe des Erblassers, § 1931 BGB, ohne von inm abzustammen.

[31] Von der Erbenstellung in gleicher Weise zu unterscheiden ist der Begriff Herkunft. Er bezieht sich auf die ständisch-soziale Abstammung und Verwurzelung (vgl. BVerfG, Beschluss vom 25. Mai 1956 - 1 BvR 83/56 -, BVerfGE 5, 17, 22, juris). Wesentlicher Regelungsgrund ist die persönlichkeitsprägende Bedeutung der örtlichen Umgebung während Kindheit und Jugend (vgl. für Art. 3 Abs. 3 GG dazu Nußberger, in: Sachs, 9. Aufl. 2021, GG Art. 3 Rn. 297 ff.), die zur diskriminierungsgefährdeten "Fremdheit" in anderer Umgebung führt. Auf die so verstandene Herkunft und Stellung des Beschwerdeführers stellt die angegriffene Norm ebenfalls nicht ab.

[32] bb) Die Nichtberücksichtigung der Erben nach Carl Erdmann Graf von Pückler knüpft nicht an ihre Abstammung an. Kriterium ist allen die Erbfolge nach Adrian Graf von Pückler.

[33] b) Die Regelung des $\S 7$ Abs. 1 Satz 2 SFPMG verstößt gegen das allgemeine Gleichbehandlungsgebot aus Art. 12 Abs. 1 Satz 1 und Satz 2 LV.

[34] aa) Gemäß Art. 12 Abs. 1 Satz 1 LV sind alle Menschen vor dem Gesetz gleich. Art. 12 Abs. 1 Satz 2 LV untersagt der öffentlichen Gewalt jede Willkür und jede sachwidrige Ungleichbehandlung. Der Gesetzgeber hat im Rahmen seiner grundsätzlich weitge- 
henden Gestaltungsfreiheit die Vergleichspaare und Sachverhalte, an denen er die Lösung seiner gesetzgeberischen Aufgabe orientiert und an die er dieselbe Rechtsfolge knüpft, die er also im Rechtssinn als gleich ansehen will, zu definieren und auszuwählen (vgl. Beschlüsse vom 16. Dezember 2010 - VfGBbg 18/10 - und vom 9. September 2016 - VfGBbg 13/16 -, https://verfassungsgericht.brandenburg.de, m.w.N.; BVerfG, Beschluss vom 21. Mai 1974 - 1 BvL 22/71, 1 BvL 21/72 -, Rn. 124, juris). Nicht nur bei belastenden Regelungen, sondern auch bei der Einräumung von Begünstigungen ist der Gesetzgeber hinsichtlich des Kreises der angesprochenen Adressaten an das Gleichbehandlungsgebot aus Art. 12 Abs. 1 Satz 1 und Satz 2 LV gebunden (vgl. für Art. 3 Abs. 1 GG: BVerfG, Beschluss vom 8. Juni 2004 - 2 BvL 5/00 -, BVerfGE 110, 412, 431, m.w. N., www.bverfg.de).

[35] Eine Ungleichbehandlung wesentlich gleicher Sachverhalte stellt hierbei allerdings nicht zwingend eine Verletzung des Gleichheitssatzes aus Art. 12 Abs. 1 Satz 1 und Satz 2 LV dar. Eine Norm verletzt Art. 12 Abs. 1 Satz 1 und Satz 2 LV, wenn durch sie eine Gruppe von Normadressaten im Vergleich zu anderen Normadressaten verschieden behandelt wird, obwohl zwischen beiden Gruppen keine Unterschiede von solcher Art und solchem Gewicht bestehen, dass sie die ungleiche Behandlung rechtfertigen können (vgl. für Art. 3 Abs. 1 GG: BVerfG, Beschluss vom 7. Mai 2013 - 2 BvR 909/06, 2 BvR 1981/06, 2 BvR 288/07 -, Rn. 76, www.bverfg.de). Der Gesetzgeber muss hinsichtlich der Verteilung der Belastungen oder Begünstigungen auf die Adressatengruppen ein folgerichtiges Konzept verfolgen (vgl. BVerfG, Beschluss vom 21. Juli 2010 - 1 BvR 611/07, 1 BvR 2464/07 -, Rn. 80, www.bverfg.de). Dies ist anhand der Umstände des jeweiligen Einzelfalls zu beurteilen (vgl. für Art. 3 Abs. 1 GG: BVerfG, Beschluss vom 8. Juni 2004 - 2 BvL 5/00 -, BVerfGE 110, 412, 432, m.w.N., www.bverfg.de). Der normative Gehalt der Gleichheitsbindung erfährt seine Präzisierung jeweils im Hinblick auf die Eigenart des zu regelnden Sachbereichs. Entscheidend ist demnach, ob für eine am Gerechtigkeitsgedanken orientierte Betrachtungsweise die tatsächliche Gleichheit oder Ungleichheit in dem jeweils in Betracht kommenden Zusammenhang so bedeutsam ist, dass der Gesetzgeber sie bei seiner Regelung beachten muss (vgl. Beschluss vom 16. Dezember 2010 - VfGBbg 18/10 -, https://verfassungsgericht.brandenburg.de).

[36] bb) Ein Konzept, das folgerichtig alle Eigentümer gleich begünstigt und nicht einzelne Eigentümer gleichheitswidrig von der Begünstigung ausschließt, lässt der Gesetzgeber aber vermissen. Hierbei steht außer Frage, dass es dem Landtag als Normgeber grundsätzlich frei steht, den leihgebenden Eigentümern der an die Erbengemeinschaft nach Theodora Gräfin von Pückler zurückübertragenen 4.673 beweglichen Vermögensgegenstände der Pückler-Callenberg-Bibliothek Branitz und weiteren 1.746 beweglichen Vermögensgegenstände das Recht auf Entsendung eines Mitglieds in den Stiftungsrat einzuräumen.

[37] Legislativ könnte somit grundsätzlich der Erbengemeinschaft nach Theodora Gräfin von Pückler das Recht eingeräumt werden, in der Stiftung mitzuwirken. Die Stellung der
Erbengemeinschaft als Eigentümerin und Leihgeberin wesentlicher Kulturgüter und Archivalien stellt gegenüber allen anderen Interessierten einen sachgerechten Grund im Sinne des Art. 12 Abs. 1 LV dar. Das Konzept, alle Mitglieder der Eigentümer-Erbengemeinschaft nach Theodora Gräfin von Pückler zur Entsendung zu berechtigen, wurde vom Gesetzgeber allerdings nicht verfolgt. Zwar hat er laut der Gesetzesbegründung (LTDrs. 6/7369 S. 5 f.) Kenntnis davon, dass die erhalten gebliebenen Kunst- und Kulturgüter, die Pückler-Callenberg Bibliothek und Archivalien des Gutsarchivs an die aus sieben Mitgliedern bestehende Erbengemeinschaft zurückübertragen wurden. Er berechtigt diese jedoch nicht als Ganzes zur Entsendung in den Stiftungsrat, sondern greift eine Gruppe von Miterben heraus.

[38] Cc) Indem gemäß § 7 Abs. 1 Satz 2 SFPMG nur die Erben nach Adrian Graf von Pückler das Recht erhalten, einen Vertreter oder eine Vertreterin aus ihrem Kreis in den Stiftungsrat zu entsenden, werden die übrigen Erben nach Theodora Gräfin von Pückler, nämlich die Erben nach Carl Erdmann Graf von Pückler, von dem Recht, einen Vertreter oder eine Vertreterin in den Stiftungsrat zu entsenden, ausgeschlossen. Die Erben nach Carl Erdmann Graf von Pückler werden durch die Regelung gegenüber den Erben nach Adrian Graf von Pückler benachteiligt. Sie sind im Gegensatz zu den Erben nach Adrian Graf von Pückler von der Möglichkeit, einen Vertreter in den Stiftungsrat zu entsenden, ausgeschlossen. Hierdurch ist auch der Beschwerdeführer benachteiligt, da er zum Kreis der von der Begünstigung ausgeschlossenen Erben gehört.

[39] Indem der Gesetzgeber in § 7 Abs. 1 Satz 2 SFPMG das Recht auf Entsendung in den Stiftungsrat an die Erbenstellung nach Adrian Graf von Pückler anknüpft, greift er aus der EigentümerErbengemeinschaft Mitglieder heraus, die kein besonderes oder gesteigertes Recht an den der Stiftung geliehenen Kulturgütern, die im Gesamthandseigentum der Erbengemeinschaft nach Theodora Gräfin Pückler stehen, haben. An diese Gruppe lässt sich kein sachlich differenzierender Grund anknüpfen, der eine besondere Stellung in Abgrenzung zu den übrigen Erben begründen könnte.

[40] dd) Die Erbengemeinschaft nach Adrian Graf von Pückler ist nicht Leihgeber weiterer privater Objekte neben den zurückübertragenen Vermögensgegenständen. Sofern der verstorbene Sylvius Hermann Graf von Pückler Objekte aus seinem Privateigentum dauerhaft oder zeitweilig an die Stiftung zu Ausstellungszwecken verliehen hat, knüpft die durch $\S 7$ Abs. 1 Satz 2 SFPMG begünstigte Eigenschaft als Erbe nach Adrian Graf von Pückler nicht an dieses Kriterium an. $\S 7$ Abs. 1 Satz 2 SFPMG gründet nicht auf der Erbenstellung nach dem sein Privateigentum verleihenden Sylvius Hermann Graf von Pückler. Die Vorschrift knüpft formal ausschließlich an die Erbenstellung nach Adrian Graf von Pückler an.

[41] ee) Das Differenzierungskriterium der Erbenstellung nach Adrian Graf von Pückler lässt sich auch nicht mit einem alternativen sachgerechten Grund im Rahmen des allgemeinen Gleichheits- 
satzes des Art. 12 Abs. 1 Satz 1 und Satz 2 LV gleichsetzen, der die vorgenommene Differenzierung innerhalb des Kreises der Mitglieder der leihgebenden Erbengemeinschaft nach Theodora Gräfin von Pückler gleichsam deckungsgleich erfassen würde.

[42] aaa) Wenn der Gesetzgeber das besondere Engagement des Sylvius Hermann Graf von Pückler als eigentlichen Anknüpfungspunkt für das Recht sieht, dass die Erben nach Adrian Graf von Pückler berechtigt sein sollen, eine Vertreterin oder einen Vertreter in den Stiftungsrat zu entsenden, könnte dieses nur ein sachgerechtes Kriterium für das Recht des Sylvius Hermann Graf von Pückler selbst sein, dem Stiftungsrat anzugehören. Das Interesse des Sylvius Hermann Graf von Pückler, seinen Geschwistern, ihren Erben, seiner Ehefrau, seinem Sohn und weiteren Nachkommen und Erben seinen Sitz im Stiftungsrat zu „vererben", ist kein sachgerechtes Kriterium für eine Differenzierung zu den übrigen Mitgliedern der Erbengemeinschaft. Denn es knüpft nicht mehr an die persönliche Leistung des Sylvius Hermann Graf von Pückler an, die ursprünglich durch das Angebot des Sitzes im Stiftungsrat der kommunalen Stiftung anerkannt werden sollte.

[43] bbb) Es ist auch keine Regelung gewählt worden, die es der Stiftung ermöglicht, besonders verdiente Mitglieder der Erbengemeinschaft nach Theodora Gräfin von Pückler in den Stiftungsrat zu berufen. Mögen im Kreis der durch § 7 Abs. 1 Satz 2 SFPMG Begünstigten auch weitere Mitglieder der Erbengemeinschaft sein, die sich für die Stiftung engagiert haben, ist das gewählte Differenzierungskriterium der Erbenstellung nach Adrian Graf von Pückler nicht deckungsgleich mit einem auf das persönliche Engagement für die Stiftung abstellenden Kriterium. Leihgaben an die Stiftung aus zurückgekauftem Privatbesitz des Sylvius Hermann Graf von Pückler könnten allenfalls eine Begünstigung seiner Witwe, seines Sohnes und weiterer Nachkommen und Erben rechtfertigen, nicht jedoch seiner Geschwister und deren Nachkommen, wobei der Wert dieser privaten Leihgaben im Verhältnis zum Wert des Eigentums der Erbengemeinschaft nach Theodora Gräfin von Pückler (4.673 bewegliche Vermögensgegenstände der Pückler-Callenberg-Bibliothek und weitere 1.746 bewegliche Vermögensgegenstände) dahingehend bewertet und gewichtet werden müsste, ob er eine Bevorzugung der privaten Einzelleihgeber durch einen Sitz im Stiftungsrat vor der leihgebenden Erbengemeinschaft überhaupt rechtfertigen könnte.

[44] ccc) Das Kriterium, dass Sylvius Hermann Graf von Pückler und seine Geschwister im Schloss Branitz gelebt haben, ermöglicht unabhängig von seiner gerügten Tragfähigkeit keine sachlich gerechtfertigte Differenzierung, da auch die Mutter des Beschwerdeführers auf Schloss Branitz gelebt hat.

[45] ddd) Innerhalb der Eigentümer-Erbengemeinschaft gibt es auch keine 10/12-Mehrheits-Erbengemeinschaft als solche, an die sich gesonderte Rechte anknüpfen ließen. Es kommt daher nicht darauf an, ob hier einzelne Rechte übertragen wurden oder über Erbanteile als Ganzes gemäß § 2033 BGB verfügt worden ist und diese einzelnen Miterben angewachsen sind.
[46] eee) Dass der Beschwerdeführer seinerseits nur einen Anteil von einem Zwölftel an der Erbengemeinschaft nach Theodora Gräfin von Pückler hält, stellt kein sachgerechtes Kriterium dar, ihn von dem Recht ausschließen, einen Vertreter der leihgebenden Erbengemeinschaft nach Theodora Gräfin von Pückler in den Stiftungsrat zu entsenden oder selbst entsandt zu werden. Der geringere Erbanteil des Beschwerdeführers rechtfertigt keine typisierende, generalisierende oder pauschalierende Betrachtungsweise, denn diese ist angesichts der Repräsentanz der Eigentümer-Erbengemeinschaft im Stiftungsrat, die mit der Vorschrift geregelt werden soll, nicht sachgerecht. Gemäß § 2032 Abs. 1 BGB ist die Erbschaft Gesamthandsvermögen. Gemäß § 2038 Abs. 1 Satz 1 BGB steht die Verwaltung des Nachlasses den Erben gemeinschaftlich zu. An den zurückübertragenen Sammlungen gehört „allen alles". Es gibt keinen nicht zu berücksichtigenden oder minderwertigen Erbanteil, nur weil er geringer ist. Zudem war zunächst ein Drittel der Erbengemeinschaft von dem gesetzlichen Ausschluss in $\S 7$ Abs. 1 Satz 2 SFPMG betroffen. Durch den zwischenzeitlichen Aufkauf von Erbanteilen wird der Inhaber eines kleinen Erbanteils nicht entrechtet. Die Gesamthandsregelungen sollen gerade den Inhaber eines kleinen Anteils vor Verfügungen durch den Inhaber eines großen Anteils schützen.

[47] fff) Das Argument, Erbstreitigkeiten sollten nicht in den Stiftungsrat getragen werden, ist ebenfalls kein tragfähiges sachgerechtes Differenzierungskriterium. Die Funktionsfähigkeit des Stiftungsrats wird durch eine Stimme nicht beeinträchtigt. Eine Blockade von Entscheidungen kann aufgrund des Mehrheitsprinzips bei der Abstimmung nicht eintreten.

[48] c) Der Ausschluss des Beschwerdeführers von der den Erben nach Adrian Graf von Pückler in der Regelung des $\S 7$ Abs. 1 Satz 2 SFPMG gewährten Begünstigung, ein Mitglied in den Stiftungsrat zu entsenden, ist nicht durch sachgerechte Differenzierungskriterien im Sinne des Art. 12 Abs. 1 LV gerechtfertigt. Das gesetzgeberische Konzept, nur Erben nach Adrian Graf von Pückler ein Entsenderecht in den Stiftungsrat einzuräumen, ist hinsichtlich der möglichen Gruppen, an die eine Differenzierung im Sinne des allgemeinen Gleichheitssatzes aus Art. 12 Abs. 1 LV sachgerecht angeknüpft werden könnte, nicht folgerichtig.

\section{C.}

[49] Da die Regelung des $\S 7$ Abs. 1 Satz 2 SFPMG gegen den allgemeinen Gleichheitssatz aus Art. 12 Abs. 1 Satz 1 und Satz 2 LV verstößt und den Beschwerdeführer benachteiligt, ist sie gemäß § 50 Abs. 4 Satz 1 VerfGGBbg für nichtig zu erklären.

[50] Die Entscheidung über die Erstattung der Auslagen beruht auf § 32 Abs. 7 Satz 1 VerfGGBbg.

D.

[51] Der Beschluss ist einstimmig ergangen. Er ist unanfechtbar. 